Communications in Physics, Vol. 24, No. 1 (2014), pp. 21-34

\title{
A NEW SOLUTION TO THE STRUCTURE EQUATION IN NONCOMMUTATIVE SPACETIME
}

\author{
NGUYEN AI VIET \\ Information Technology Institute, Vietnam National University, Hanoi, Vietnam \\ E-mail: naviet@vnu.edu.vn \\ Received 31 December 2013 \\ Accepted for publication 17 February 2014
}

\begin{abstract}
In this paper, starting from the common foundation of Connes' noncommutative geometry (NCG) [1-4], various possible alternatives in the formulation of a theory of gravity in noncommutative spacetime are discussed in details. The diversity in the final physical content of the theory is shown to be the consequence of the arbitrary choices in each construction steps. As an alternative in the last step, when the structure equations are to be solved, a minimal set of constraints on the torsion and connection is found to determine all the geometric notions in terms of the metric. In the Connes-Lott model of noncommutative spacetime, in order to keep the full spectrum of the discretized Kaluza-Klein theory [8], it is necessary to include the torsion in the generalized Einstein-Hilbert-Cartan action.
\end{abstract}

Keywords: non-commutative geometry, gravity, general relativity, Kaluza-Klein theory, Cartan formalism.

\section{INTRODUCTION}

More than twenty years after A.Connes [1-3] suggested the idea of using NCG in physics, the contruction of the physical theory of gravity in noncommutative spacetime has not reached a completely satisfactory state. The basic ideas of generalizing Riemannian geometry in noncommutative context have been presented in [4-9]. It was Chamseddine and Fröhlich who initiated the first real effort to construct an extended Einstein theory of gravity utilizing the noncommutative geometric model of Connes-Lott's two- sheeted spacetime [10]. Following this pioneering paper, a great number of other papers have discussed different aspects of this interesting topic. However, these works do not agree on the physical content of the final action, where the physical fields make their appearance. This is why the most interesting application of the Connes-Lott's spacetime could not find the final physical content. In this paper, we will try to clarify this topic in a more systematic way by reconsidering all the possible assumptions made in every steps of construction.

The mainstream papers, following the Chamseddine-Frölich's construction, have agreed with the field content of only the standard Einstein metric field along with a Brans-Dicke scalar, which represents the distance between pairs of points on the two separate sheets. However, in several other works [11], with a different formulation of the action, the scalar field does not have 
a kinetic term, so it would not be present in the final theory as a physical field. That is to say noncommutative spacetime could not bring a new physical content. Intuitively, it is hard to imagine what forces us to measure the distance between two points on different sheets of spacetime with just one metric.

Some alternative constructions of our group [8,9,12-17] suggest that the gravity theory of the Connes-Lott's spacetime might have a Kaluza-Klein spectrum, which contains massless tensor, vector and scalar fields together with their massive counterparts in a harmonic expansion in the discrete internal spacetime.

The reason for this diversity is due to the fact that although most papers have accepted Connes'construction as the common foundation, a unique recipe to build the final physical theory is still lacking. With Connes' spectral triple given, there is still a considerable amount of arbitrariness at every stage of the construction of the final action leading to different final results.

The purpose of this paper is to point out the alternative choices at various stages of the theoretical construction and find a new minimal set of constraints on the torsion and connection to complete the Cartan structure equations.

This set is "minimal" in the sense that it does not place any further constraints on the initially postulated metric structure. In fact, as seen in this paper, the too strict constraints have abandoned a lot of interesting physical contents from the theory in the original constructions.

Finally, based on some physical considerations, a new solution to the structure equations is found leading to the final action, where both torsion and curvature contribute to a full self consistent action.

\section{THE GENERAL CONSTRUCTION PROCEDURE}

Let us summarize the general construction procedure to construct the most general theory of gravity following [18-21]. The most general construction procedure for a theory of gravity having both torsion and curvature has the following steps:

Step 1: The choice of 0-forms and the differential operator. The Einstein's theory of general relativity is intimately built on the algebra of real functions as 0 -forms and the differential operator $d=d x^{\mu} \partial_{\mu}$. With such choices, the 1-forms are automatically hermitian, since the differential operator is hermitian with the complex conjugate operator $*$. Alternatively, one can build the theory on the algebra of complex functions as 0 -forms. However, one must restrict the algebra of the hermitian 1-form. To define the hermiticity, one must define a $*$ operation. In a more general context, the $*$ operator can be generalized to be the involutive operation in different ways, which are not necessarily related to the complex conjugate operator.

Step 2: The formulation of the equivalence principle and the definition of 1-forms in different reference frame. The general reference frame is defined in terms of the basis $d x^{\mu}$, which mean that any 1-form a can be expressed as $\mathbf{a}=d x^{\mu} a_{\mu}$ where $a_{\mu}$ are real functions. The Equivalence Principle of Einstein states that there exists an local orthonormal reference frame with the basis $e^{a}$ which is related to the general one $e^{a}=d x^{\mu} e_{\mu}^{a} ; d x^{\mu}=e^{a} e_{a}^{\mu}$. The gravity is contained in the vierbein function $e_{a}^{\mu}$, which defines the metric $g_{\mu \nu}=\eta^{a b} e_{\mu}^{a} e_{\nu}^{b}$ where $\eta^{a b}=\operatorname{diag}(1,-1,-1,-1)$. In the spinor representation widely used in physics, the bases $d x^{\mu}$ and $d x^{a}$ can be represented by the Dirac matrices $\gamma^{\mu}$ and $\gamma^{a}$. In this case, the metric is determined by the trace formula $g_{\mu \nu}=\frac{1}{4} \operatorname{tr}\left\{\gamma_{\mu}, \gamma_{\nu}\right\}$ 
Step 3: The definition of 2-forms via an wedge product. The algebra of 2-forms can be generated by the antisymmetric wedge products $d x^{\mu} \wedge d x^{\nu}=-d x^{\nu} \wedge d x^{\mu}$. The antisymmetry is required to avoid the "junk" forms, which is zero and having a non-zero derivative. Once the wedge product is defined, the algebra of $n$-forms can be also defined.

Step 4: The formulation of the structure equations and their solutions. In the Cartan's formulation, the Riemannian geometry can be constructed in the most general context with the notions of connection, curvature and torsion defined in the following structure equations

$$
\begin{gathered}
r_{b}^{a}=d \omega_{b}^{a}+\omega_{c}^{a} \wedge \omega_{b}^{c} \\
t^{a}=d e^{a}-e^{b} \wedge \omega_{b}^{a}
\end{gathered}
$$

In the general Cartan's formulation, the metric (vierbein) and connection are independent basic geometric notions, while the curvature and torsion can be derived from the basic geometric notions.

There are some speculations that the Riemannian geometry with a non-vanishing torsion can describe the gravity of the spin matter. In the case of vanishing torsion, the second structure equation becomes the torsion free condition

$$
t^{a}=d e^{a}-e^{b} \wedge \omega_{b}^{a}=0
$$

which can be used to determined the connection in terms of the metric (vierbein). The theory then reduces to the Christoffel-Levi-Civita's formulation of the ordinary Riemannian geometry. In this theory, the curvature can be expressed in terms of the Christoffel symbols, which are given in terms of the metric. The Riemannian geometry in this case, as shown by Einstein, describes the gravity.

Step 5: The final Einstein-Hilbert-Cartan action is given by the Lagrangian

$$
L=\frac{1}{8 \pi G_{N}} r+\frac{1}{G_{T}^{2}}<t_{a}, t^{a}>+\mathcal{L}_{\text {matter }},
$$

where $G_{N}$ and $G_{T}$ are the ordinary Newton and torsion related coupling constants; $\mathcal{L}_{\text {matter }}$ is the Lagrangian of the matter fields in the generalized Riemannian spacetime. Applying the variation principle to minimize the above action, one can receive the generalize Einstein equation. The solutions of the Einstein equation will describe the gravity and the matter fields. When $\mathcal{L}_{\text {matter }}=0$ the solutions of the Einstein equation will describe possible spacetime structure with pure gravity.

\section{THE COMMON FOUNDATION: CONNES' FORMULATION}

There is extensive literature about Connes' general formalism of NCG [1-7, 9, 22-26].

Here, for the sake of completeness, we shall begin with a brief review of the relevant ideas that are necessary for this paper following the original idea of A.Connes [1,2]. In the construction of the gravity theory, we will follow the parallel steps with the ones presented in Sec. II. More detailed formulas can be found in our papers [8,12-17].

In Connes' formulation, the basic building block of noncommutative geometry is the spectral triple $(\mathcal{A}, D, \mathcal{H})[1]$ as follows:

i) The algebra $\mathcal{A}$ of the 0 -forms replaces the functions in a generalized Gelfand's construction. The replacement of the number fields by the algebra $\mathcal{A}$ leads to the concept of $\mathcal{A}$-bimodule as a generalization of the vector space. 
ii) The Dirac operator $D$ acts on $F \in \mathcal{A}$ giving a subset of the $\mathcal{A}$-bimodule of 1-forms. Therefore, $D F$ can be formally written as

$$
D F=\sum_{M}\left(D F^{M}\right)(D F)_{M}, M \in \alpha, F^{M} \in \mathcal{A}
$$

where $\alpha$ is a finite set. That is to say, the Eqn.(5) postulates the existence of a finite basis $\left(D F^{M}\right)$ of the set $\Omega^{1}(\mathcal{A})$ of 1 -forms. In other words, $\Omega^{1}(\mathcal{A})$ has an algebraic structure of finite projective $\mathcal{A}$-bimodule. The elements $\left(D F^{M}\right)$ are also given by the Dirac operator acting on $F^{M} \in \mathcal{A}$.

The non-commutativity of the theory does not necessarily come from the non-commutativity of the algebra $\mathcal{A}$. For example, the Connes-Lott two-sheeted noncommutative space-time model [3] is essentially based on the commutative algebra $C^{\infty}(C, \mathcal{M}) \bigoplus C^{\infty}(C, \mathcal{M})$, where $\mathcal{M}$ is the usual physical spacetime. It is the Dirac operator as an outer automorphism that brings about the noncommutativity. Outer automorphism is the property of an operator, whose action on an element gives an element outside of the initial domain of the elements.

It is possible to extend the definition of $D$ onto the bimodule of 1 -forms $\Omega^{1}(\mathcal{A})$ and define the product of 1 -forms in order to build the $\mathcal{A}$-bimodule $\Omega^{2}(\mathcal{A})$ of 2 -forms. This procedure can be repeated to construct the universal algebra $\Omega^{*}(\mathcal{A}) \equiv \bigoplus_{p} \Omega^{p}(\mathcal{A})$ of differential forms on the algebra $\mathcal{A}$ with the Dirac operator $D$. An involutive operation on $\mathcal{A}$ can be extended uniquely to the one on the algebra $\Omega^{*}(\mathcal{A})$. that takes

The universal envelope algebra $\Omega^{*}(\mathcal{A})$ has a graded structure with the Dirac operator $D$

$$
\begin{gathered}
c c c D: \Omega^{p} \longrightarrow \Omega^{p+1} \\
D\left(\left(D F_{1}\right) \ldots\left(D F_{p}\right) F\right) \equiv(-1)^{-1}\left(D F_{1}\right) \ldots\left(D F_{p}\right)(D F), \forall F, F_{1}, \ldots, F_{p} \in \Omega^{p}(\mathcal{A}),
\end{gathered}
$$

which implies

$$
\begin{array}{cccc}
D^{2} F & = & 0, & \forall p, F \in \Omega^{*}(\mathcal{A}) \\
D\left(F_{1} F_{2}\right) & = & \left(D F_{1}\right) F_{2}+(-1)^{\operatorname{deg} F_{1}} F_{1}\left(D F_{2}\right), & \forall F_{i} \in \Omega^{*}(\mathcal{A})
\end{array}
$$

iii) The Hilbert space $\mathcal{H}$ is where the elements of $\mathcal{A}$, differential forms and exterior derivative act as operators. In the Connes-Lott's model, it is chosen as the direct sum of the Hilbert spaces of left-handed and right-handed spinors $\mathcal{H}=\mathcal{H}_{L} \bigoplus \mathcal{H}_{R}$.

The representation of the operators corresponding to differential forms on a given Hilbert space is realized by the graded, involution preserving homomorphism $\pi$,

$$
\begin{array}{ccc}
\pi & : & \Omega^{*}(\mathcal{A}) \longrightarrow \mathcal{L}(\mathcal{H}) \\
\pi_{p}\left(\left(D F_{1}\right) \ldots\left(D F_{p}\right) F\right) & = & \prod_{i=1}^{p}\left[D, \pi_{0}\left(F_{i}\right)\right] \pi_{0}(F),
\end{array}
$$

where $\mathcal{L}(\mathcal{H})$ denotes the space of bounded operators on the Hilber space $\mathcal{H}$ and $\pi_{0}$ is a representation of the algebra $\mathcal{A}$ on $\mathcal{H}$. Henceforth, the symbol $D$ will denote both the Dirac operator $D$ and its representation as a self-adjoint operator. It is assumed that $D$ has a compact resolvent, such that the commutator $\left[D, \pi_{0}(F)\right]$ is also a bounded operator $\forall F \in \mathcal{A}[1]$.

It is possible to choose a finite basis $\left(D F^{M}\right)$ as in Eqn.(5) and represent it by $\Theta^{M}=$ $\pi_{1}\left(\left(D F^{M}\right)\right)$. Therefore, an arbitrary 1-form $\omega \in \Omega^{1}(\mathcal{A})$ is represented as

$$
U=\pi_{1}(\omega)=\Theta^{M} U_{M} .
$$


The Dirac operator is represented in the "general" basis $\Theta^{M}$ as

$$
\begin{aligned}
D & =\Theta^{M} D_{M} \\
(D F)_{M} & \equiv D_{M} F=\left[D, \pi_{0}\left(F_{M}\right)\right] .
\end{aligned}
$$

With the differential forms in hand, one can follow the standard procedure to introduce a metric structure, the connection 1-forms, the torsion and, the curvature 2-forms to construct a theory of gravity. The Cartan's structure equations can be formulated and solved with some additional constraints to express all the geometric quantities in terms of the metric. The physical Einstein-Hilbert-Cartan action can be built from the curvature and torsion.

The formal procedure is rather well defined. Nevertheless, in a given specific representation, there are still alternate steps that lead to diverse physical contents of the final theory of gravity. In the next section, we will follow the steps in formulating the theory by and point out the most general theories and its possible alternatives as special cases.

\section{ALTERNATIVES IN THE THEORY CONSTRUCTION}

\section{IV.1. The choice of the involutive operation and its subalgebra}

In the Connes-Lott model [3], which concerns mainly with the Standard Model, the physical fields are not represented by the most general 1-forms. Instead, only the hermitian 1-forms are considered as physical. Therefore, the involutive operation as the hermitian conjugate $*$ is introduced in the bimodule of 1 -forms. In such a construction, the 0 -forms continue to be complex, while only hermitian 1-forms are relevant for physical purposes. The hermitian forms do not form a $\mathcal{A}$-bimodule, but rather as a bimodule of a subalgebra of $\mathcal{A}$, which is the algebra of real functions $\mathcal{B}=C^{\infty}(R, \mathcal{M}) \bigoplus C^{\infty}(R, \mathcal{M})$.

It suggests that a subalgebra of $\mathcal{A}$ maybe sufficient to construct a gauge theory with the same physical content.

In one of our papers [15], it is shown that, the Standard Model can be constructed in the context of NCG, if one chooses the subalgebra $\mathcal{A}^{\prime} \in \mathcal{A}$ of the 0 -forms in the basic spectral triple as follows

$$
F=\left(\begin{array}{cc}
f(x) & 0 \\
0 & f^{*}(x)
\end{array}\right), f(x) \in C^{\infty}(C, \mathcal{M})
$$

where $f^{*}(x)$ is the complex conjugate of $f(x)$.

The complex conjugate will be coincident with the involutive operation " " used in a series of papers $[8,13-15]$,

$$
\tilde{F}=F^{*}=\left(\begin{array}{cc}
f^{*}(x) & 0 \\
0 & f(x)
\end{array}\right)
$$

It is noted that the restriction to the subalgebra $\mathcal{A}^{\prime}$ is dicted by Physics. In fact, in a more general context, one can start with the algebra $\mathcal{A}$ with the involutive operation defined as follows

$$
F=\left(\begin{array}{cc}
f_{1}(x) & 0 \\
0 & f_{2}(x)
\end{array}\right), \tilde{F}=\left(\begin{array}{cc}
f_{2}(x) & 0 \\
0 & f_{1}(x)
\end{array}\right), f_{1}(x), f_{2}(x) \in C^{\infty}(C, \mathcal{M}) .
$$

It is shown in [15] that in order to have correct kinetic terms for all the fields, one must restrict all the 0 -forms and all coefficients of higher differential forms subjected to the algebra $\mathcal{A}^{\prime}$. The universal envelope algebra $\Omega^{*}\left(\mathcal{A}^{\prime}\right)$ constructed with the algebra $\mathcal{A}^{\prime}$ can be used to represent the 
physical fields without any further condition.

The example of the involution " " has shown that, in general, it is possible to choose various alternative involution operations to have the desirable physical contents suitable for a given application. This alternative produces the same field contents for the gauge theory as the ConnesLott model does with a proper definition of the inner product.

\section{IV.2. The equivalence principle}

The foundation of general relativity in noncommutative spacetime is based on the equivalence principle. As traditionally stated by Einstein, this principle postulates the existence of the general and the locally orthonormal frames, which can be transformed into each other by a local orthonormal invertible transformation. The general frame consists of any finite basis of the module of generalized 1-forms as formulated in Sec. II. The exterior of derivatives of forms are defined in this frame. Therefore, the differential calculus is always carried out in this frame and the results will be transformed into other frames if necessary. On the other hand, the local orthonormal frame has the advantage in the algebraic calculations of forms at the same location. Therefore, it is the most convenient basis for the formulation of the structure equations for the connection, torsion and curvature.

In general, let us denote the basis of the general frame as $\Theta^{M}$ and the basis of the local orthonormal frame as $\Theta^{A}$. The generalized vielbein is the spacetime dependent transformation:

$$
\begin{aligned}
\Theta^{M} & =\Theta^{A} E_{A}^{M}(x) \\
\Theta^{A} & =\Theta^{M} E_{M}^{A}(x),
\end{aligned}
$$

where the 0 -forms $E_{M}^{A}(x)$ and $E_{A}^{M}(x)$ are inverses of each other,

$$
\begin{aligned}
& E_{A}^{M}(x) E_{N}^{A}(x)=\delta_{N}^{M} \\
& E_{M}^{A}(x) E_{B}^{M}(x)=\delta_{B}^{A} .
\end{aligned}
$$

Since the derivatives are defined only in the "general" frame, the Dirac operator $D$ is represented in the local orthonormal basis as

$$
D=\Theta^{A} E_{A}^{M}(x) D_{M}
$$

Indeed, the metric structure is encoded in the Dirac operator via the presence of the vielbein $E_{A}^{M}(x)$. The situation is exactly the same as in the Einstein's General Relativity.

The structure of $E_{A}^{M}(x)$ is where different choices are possible. In the most general case, each 0-form $E_{M}^{A}$ contains a pair of the usual functions.

For the sake of definiteness, let us take the example in the Connes-Lott's two-sheeted spacetime model, which is generally referred to by most of the authors. In this model, the 0 -forms and therefore the vielbeins $E_{M}^{A}(x)$ and $E_{A}^{M}(x)$ where $M=\mu, 5$ and $A=a, \dot{5}$ are represented as $2 \times 2$ matrices $[8,10,12,13,15]$. Specially, in the most general form, the vielbeins are given as

$$
\begin{aligned}
E_{a}^{\mu}(x) & \equiv\left(\begin{array}{cc}
e_{1 a}^{\mu}(x) & 0 \\
0 & e_{2 a}^{\mu}(x)
\end{array}\right), \quad E_{\dot{5}}^{\mu}(x) \equiv 0, \\
E_{a}^{5}(x) & \equiv-\left(\begin{array}{cc}
a_{1 a} & 0 \\
0 & a_{2 a}
\end{array}\right) \equiv-A_{a}(x)=-E_{a}^{\mu} A_{\mu},
\end{aligned}
$$




$$
E_{\dot{5}}^{5}(x) \equiv\left(\begin{array}{cc}
\phi_{1}^{-1}(x) & 0 \\
0 & \phi_{2}^{-1}(x)
\end{array}\right) \equiv \Phi^{-1}(x),
$$

where $e_{1,2 a}^{\mu}(x)$ are two different vierbeins on the two sheets of space-time. Similarly, $a_{1,2}(x)$ and $\phi_{1,2}(x)$ are respectively vector and scalar fields. The vielbein $E_{\dot{5}}^{\mu}(x)$ can always be chosen as zero without any loss of generality because of the residue rotational arbitrariness.

The vielbeins $E_{A}^{M}$ are invertible as follows,

$$
\begin{gathered}
E_{\mu}^{a}(x) \equiv\left(\begin{array}{cc}
e_{1 \mu}^{a}(x) & 0 \\
0 & e_{2 \mu}^{a}(x)
\end{array}\right), \quad E_{5}^{a}(x) \equiv 0 \\
E_{\mu}^{\dot{5}}(x) \equiv A_{\mu}(x) \Phi(x), \quad E_{5}^{\dot{5}}(x) \equiv\left(\begin{array}{cc}
\phi_{1}(x) & 0 \\
0 & \phi_{2}(x)
\end{array}\right) \equiv \Phi(x) .
\end{gathered}
$$

The Dirac operator is

$$
D=\left(\begin{array}{cc}
\gamma^{a} e_{1 a}^{\mu} \partial_{\mu} & -m \gamma^{a} e_{1 a}^{\mu} a_{1 \mu}+m \gamma^{5} \phi_{1} \\
\gamma^{a} e_{2 a}^{\mu} a_{2 \mu}-m \gamma^{5} \phi_{2} & \gamma^{a} e_{2 a}^{\mu} \partial_{\mu}
\end{array}\right)
$$

By imposing the constraints $e_{1 a}^{\mu}=e_{2 a}^{\mu}, a_{1 \mu}=a_{2 \mu}, \phi_{1}=\phi_{2}$, the results of our first paper [12] can be recovered: the zero-modes of the Kaluza-Klein theory are obtained. In the other investigations $[10,23,25]$ the vector field is assumed to vanish. It is worth to note that in [10], Chamseddine and Frölich have started from the most general form of vielbein, which contains tensor, vector and scalar fields. It is interesting to point out that in their construction, the local orthonormal frame has been used to carry out the derivation, which means that a additional constraint has been intimately imposed. That was why the vector fields are omitted from the theory.

At this point, we can show that without an additional assumption, the theory does not have any restrictions on the field content of the general vielbein.

Since Eqns. (17), (18) and (19) cannot give any restriction to the theory, one must look for further possibilities.

The metric is given by a definition of the inner product, which is a sesquilinear functional

$$
\begin{array}{ccc}
<,> & : & \Omega^{1}(\mathcal{A}) X \Omega^{1}(\mathcal{A}) \longrightarrow \mathcal{A} \\
<U F, V G>= & \tilde{F}<U, V>G .
\end{array}
$$

In particular, one obtains

$$
\mathcal{G}^{M N}(x)=<\Theta^{M}, \Theta^{N}>=\tilde{E}_{A}^{M}(x) \eta^{A B} E_{B}^{N}(x),
$$

directly from the othonormality of the frame $\Theta^{A}$ in the following form

$$
\mathcal{G}^{A B}(x)=<\Theta^{A}, \Theta^{B}>\equiv \eta^{A B}=\tilde{E}_{M}^{A}(x) \mathcal{G}^{M N} E_{M}^{B}(x),
$$

From Eqn.(15), that defines the inverse vielbein, one can see that the equations (21) and (22) are in fact equivalent. As these equations define the metric tensor in terms of vielbein, one can conclude that they do not give any further restriction on the vielbein.

In the article [25] an argument is made to show that the vector field is related to a gauge transformation that emerges as an internal shift in an analogy with the internal circle of the ordinary Kaluza-Klein theory. In fact, the internal space of this model consists of only two points. Hence, one cannot speak about the $U(1)$ gauge transformation in relation to the shift in the internal circle. 
The gauge invariance, if absolutely needed, must be guaranteed by a different motivation.

As proven in the article [13], the "zero-mode only" constraint of [12] is a special case of the torsion free condition when one solves the structure equation. There exist various ways to impose conditions in order to solve the structure equations. We will discuss this possibilities later.

One might also argue following [10] that in a $\Gamma$-representation, in which the bases $\Theta^{M}$ and $\Theta^{A}$ are represented as $\Gamma^{M}$ and $\Gamma^{A}$, the trace of these matrices might lead to a restriction on the vector field.

Let us consider this possibility, starting from the basis

$$
\Gamma^{a}=\gamma^{a} \bigotimes 1, \Gamma^{\dot{5}}=\gamma^{5} \bigotimes \sigma,
$$

where

$$
\begin{aligned}
\mathbf{1} & =\left(\begin{array}{cc}
1 & 0 \\
0 & 1
\end{array}\right) \\
\sigma & =\left(\begin{array}{cc}
0 & -1 \\
1 & 0
\end{array}\right)
\end{aligned}
$$

In this choice of basis, the "general" $\Gamma^{M}$ matrices are

$$
\begin{aligned}
\Gamma^{\mu} & =\left(\begin{array}{cc}
\gamma^{a} e_{1 a}^{\mu}(x) & 0 \\
0 & \gamma^{a} e_{2 a}^{\mu}(x)
\end{array}\right) \\
\Gamma^{5} & =\left(\begin{array}{cc}
-\gamma^{a} e_{1 a}^{\mu} a_{1 \mu} & \gamma^{5} \phi_{2}^{-1}(x) \\
\gamma^{5} \phi_{1}^{-1}(x) & -\gamma^{a} e_{2 a}^{\mu} a_{2 \mu}
\end{array}\right) .
\end{aligned}
$$

All the trace formulas of the $\Gamma$ matrices are consistent with the metric tensors defined in Eqns.(21) and (22). For example, the trace

$$
\begin{aligned}
\mathcal{G}^{\mu 5} & =\operatorname{Tr}\left(\Gamma^{\mu} \Gamma^{5}\right)=\tilde{E}_{A}^{\mu} \eta^{A B} E_{B}^{5}=\tilde{E}_{a}^{\mu} \eta^{a b} E_{b}^{5} \\
& =\left(\begin{array}{cc}
-e_{1 a}^{\mu} \eta^{a b} e_{1 b}^{\nu} a_{1 \mu} & 0 \\
0 & -e_{2 a}^{\mu} \eta^{a b} e_{1 b}^{\nu} a_{2 \nu}
\end{array}\right)^{2}
\end{aligned}
$$

gives the vanishing vector field only if one requires that $G^{\mu 5}$ vanishes. However, this happens only in specific cases such as in the flat spacetime. In the general frame, in fact, one can always choose the basis so that the metric $\mathcal{G}^{\mu 5}=0$. However, with such a choice, the tensor $\mathcal{G}^{A B}$ will not be constant any more.

The last possibility to have a constraint on the vielbein is to see whether all its components have kinetic terms in the final action, since without the kinetic terms, these fields will not survive as physical fields. If a field content in parallel with the Kaluza-Klein spectrum is desirable, it will be shown that, one can choose a set of minimal conditions in such a way that maximal number of fields will survive with the corresponding kinetic terms as shown in [8,13-15].

\section{IV.3. Alternative definitions of two-forms}

In order to formulate the torsion and curvature in the structure equations, one must define the $\mathcal{A}$-bimodule of 2 -forms. This module should not contain the "junk forms"( the non-vanishing forms that are differentials of the forms that are indentical to zero in the $\pi$ representation). As an illustration of a "junk form" in the usual differential geometry, let us consider the 1-form 
$\omega=d f . f-f . d f$, which is identical to zero. However, the "junk form" $d \omega=-2 d f . d f$ is nonvanishing. To eliminate these "junk forms", one defines wedge products and replaces the ordinary product with the wedge product to construct 2-forms as products or exterior derivative of 1-forms.

In NCG, one can also follow the technique of using auxialary fields $[1,6]$ to find the general form of the 2-forms, which are not "junk forms". On the other hand, in the case of ConnesLott model, one can successfully define the wedge product, which in fact eliminates the "junk forms" $[8,12,13,16,17]$.

The definition of the wedge product is not unique. In fact, one can choose a wedge product that is fully anti-symmetric as in $[8,12,13]$ for the theories of pure gravity . However, in order to have a quartic Higgs potential for the gauge fields one must choose a wedge product, where $\Gamma^{5} \wedge \Gamma^{5}$ is not zero. This arbirariness might result in additional terms in the final action.

Alternatively, in $[16,17]$ the wedge product is chosen as being fully anti-symmetric, but one can still produce a quartic Higgs potential, since the components of forms related to the internal space is characterized by two complex quantities, which are conjugates of each other, instead of one.

\section{IV.4. Alternative sets of constraints}

The torsion and curvature of Riemannian geometry can be generalized via the structure equations in the local orthonormal frame as follows

$$
T^{A}=D \Theta^{A}-\Theta^{B} \Omega_{B}^{A}
$$

and

$$
R_{B}^{A}=D \Omega_{B}^{A}+\Omega_{C}^{A} \Omega_{B}^{C},
$$

where $\Omega_{B}^{A}$ are the connection 1-forms. The structure equations are not sufficient to determine the connection, torsion and curvature in terms of metric fields. Therefore, some additional constraints on the torsion and connection must be imposed.

In ordinary Riemmanian geometry, the torsion free condition together with the metric compatibility equation completely determines the connection and curvature in terms of the metric structrure. In NCG, the torsion free condition $T^{A}=0$ has been shown to lead to the following restriction on the metric structure [13]

$$
\begin{aligned}
e_{1 a}^{\mu}(x) & =\beta(x) e_{2 a}^{\mu}(x), \\
a_{1 \mu}(x) & =\beta(x) a_{2 \mu}(x), \\
\phi_{1}(x) & =\beta(x) \phi_{2}(x),
\end{aligned}
$$

where $\beta(x)$ is a dilaton field with a hightly non-linear potential. In the special case of this restriction, where $\beta(x) \equiv 1$ one obtains the theory in [12] containing only the massless modes of the truncated Kaluza-Klein theory with the vierbein, vector and Brans-Dicke scalar fields.

Since a theory with the maximal spectrum would be interesting for broader applications, it has been our purpose to find a minimal set of constraints, which does not impose any restriction on the metric. In [8], the following set has been found

$$
\begin{array}{ccc}
T_{a b c} & = & T_{a b \dot{5}}=T_{a \dot{5} b}=0 \\
\operatorname{Tr}\left(T_{\dot{5} A B}\right) & = & 0,
\end{array}
$$


together with the metric compatible condition

$$
\begin{array}{ccc}
\Omega_{A B} & = & -\Omega_{B A} \\
\operatorname{Tr}\left(\Omega_{A B} \mathbf{r}\right) & = & 0 .
\end{array}
$$

The set of constraints (30) and (31) are direct generalizations of the spacetime torsion free and the metric compatibility conditions. The additional constraints look rather unnatural. However, it is the first model with the spectrum, which is consistent with the intuitive discretized KaluzaKlein theory. When the internal space is discretized to just two points, a harmonic expansion in the internal dimension would give gravity, vector and scalar fields in pairs with their massive excitations. In fact, all the vielbein components in the equation (17) survive in the curvature with their appropriate kinetic terms.

In the next section, we show that it is possible to find another minimal set of constraints. This illustrates the arbitrariness in choosing the alternative constraint.

\section{IV.5. The final action's formula}

With a proper set of constraints on the connection 1-forms and torsion 2-form, one can solve the first structure equation (27) to express all the components of the connections and torsion completely in terms of vielbeins. Then, the curvature is determined in terms of the connections from the second structure equation (28).

With all the geometric notions in hand, there is still an arbitrariness in the formula of the final action. One may choose the Wodzicki residue, which gives no kinetic term for the scalar field [11]. With the same vielbein, the Dixmier trace formula, on the other hand, contains the kinetic term for the scalar field [10,23]. Therefore, the decision, whether the scalar field can exist or not in the theory, depends on the choice of the definition of action. Perhaps, using the Wodzicki residue, one might have to include more characteristics into the action to retain the scalar field in the final theory.

The solution to be found in this paper shows a similar situation: the constributions from the curvature do not contain kinetic terms for the vector and scalar fields. To retain these fields in the theory, one must include the quadratic term of the torsion.

It is important to note that in the formula of action that contains the inner product, the definition of the involutive operation may also alter the action.

\section{NEW CONSTRAINTS AND NEW SOLUTION}

In order to obtain the ordinary gravity, any set of constraints must be an extension of the ordinary metric compatibility and torsion free conditions.

Let us discuss the metric compatibility condition first. The simplest way is to generalized the metric compatibility condition as

$$
\Omega_{A B}=-\Omega_{B A} \text {. }
$$

Our previous work $[8,12,13]$ uses the same form of the metric compatibility together the hermitian conjungate and a reality condition. In this work, the metric compatibility condition is consistent with the involutive operation " ", which is originally defined on 0 -forms, is just the identity on 1 -forms.

The torsion free condition $T_{A}=0$ has been proven to lead to restriction on the vielbein $[12,13]$. Therefore, we should look for a weaker condition. The most direct generalization of the 
torsion free condition is

$$
T_{a}=0 .
$$

To see whether the metric compatbility and torsion free conditions in the equations (32) and (33) are enough to solve the first structure equation, let us write its components as

$$
\begin{aligned}
T_{a b c} & =\left(D \Gamma_{a}\right)_{b c}+\frac{1}{2}\left(\Omega_{a b c}-\Omega_{a c b}\right) \\
T_{a \dot{5} b} & =\left(D \Gamma_{a}\right)_{\dot{5} b}+\frac{1}{2}\left(\Omega_{a \dot{5} b}-\Omega_{a b \dot{5}}\right) \\
T_{\dot{5} a b} & =\left(D \Gamma_{\dot{5}}\right)_{a b}+\frac{1}{2}\left(\Omega_{\dot{5} a b}-\Omega_{\dot{5} b a}\right) \\
T_{\dot{5} \dot{5} b} & =\left(D \Gamma_{\dot{5}}\right)_{\dot{5} b}+\frac{1}{2}\left(\Omega_{\dot{5} \dot{5} b}-\Omega_{\dot{5} b \dot{5}}\right) \\
T_{a b \dot{5}} & =\left(D \Gamma_{a}\right)_{b \dot{5}}+\frac{1}{2}\left(\Omega_{a b \dot{5}}-\Omega_{a \dot{5} b}\right) \\
T_{\dot{5} a \dot{5}} & =\left(D \Gamma_{\dot{5}}\right)_{a \dot{5}}+\frac{1}{2}\left(\Omega_{\dot{5} a \dot{5}}-\Omega_{\dot{5} \dot{5} a}\right) \\
T_{a \dot{5} \dot{5}} & =\left(D \Gamma_{a}\right)_{\dot{5} \dot{5}}+\frac{1}{2}\left(\Omega_{a \dot{5} \dot{5}}+\tilde{\Omega}_{a \dot{5} \dot{5}}\right) \\
T_{\dot{5} \dot{5} \dot{5}} & =\left(D \Gamma_{\dot{5}}\right)_{\dot{5} \dot{5}}+\frac{1}{2}\left(\Omega_{\dot{5} \dot{5} \dot{5}}+\tilde{\Omega}_{\dot{5} \dot{5} \dot{5}}\right) .
\end{aligned}
$$

It is obvious that the conditions (32) and (33)are not enough. There are various way to choose an additional condition. The simplest condition we can find is

$$
\Omega_{A B \dot{5}}=0
$$

With three conditions (32), (33) and (35) all the non-vanishing components of the torsion and connection are given by

$$
\begin{array}{rcc}
\Omega_{a b c}= & -\left(D \Gamma_{a}\right)_{b c}+\left(D \Gamma_{b}\right)_{a c}-\left(D \Gamma_{c}\right)_{b a} \\
\Omega_{a \dot{5} b}= & -2\left(D \Gamma_{a}\right)_{\dot{5} b} \\
\Omega_{\dot{5} a b}= & 2\left(D \Gamma_{a}\right)_{\dot{5} b} \\
T_{\dot{5} b c}= & \left(D \Gamma_{\dot{5}}\right)_{b c}+\left(D \Gamma_{b}\right)_{\dot{5} c}-\left(D \Gamma_{c}\right)_{\dot{5} b} \\
T_{\dot{5} \dot{5} b}= & \left(D \Gamma_{\dot{5}}\right)_{\dot{5} b} \\
T_{\dot{5} \dot{5} \dot{5}}= & \left(D \Gamma_{\dot{5}}\right)_{\dot{5} \dot{5}} .
\end{array}
$$

Since the exterior derivative of $\Gamma^{A}$ can be calculated in terms of vielbein, the torsion and connections are expressed completely in terms of the metric. Therefore, the second structure equation (28) determines the curvature also in term of the metric. No further restriction on the vielbein is required.

Now if all the fields in the vielbein (17) will have kinetic terms in the final action, they will survive as physical fields.

The scalar curvature is given

$$
R=<\Gamma^{A} \wedge \Gamma^{B}, R_{A B}>
$$

After explicit calculation of the scalar curvature, we find that the kinetic terms for the vector and scalar fields are not in the scalar curvature as they do in the other solution in [8]. 
To retain these fields in the theory, the contribution from the torsion must be included as the inner product

$$
<T_{A}, T^{A}>=\tilde{T}_{A B C}<\Gamma^{B} \wedge \Gamma^{C}, \Gamma^{D} \wedge \Gamma^{E}>T_{D E}^{A}
$$

Therefore the final Einstein-Hilbert-Cartan Lagrangian of our theory is

$$
\mathcal{L}=\frac{1}{16 \pi G_{N}} R+\frac{1}{G_{T}^{2}}<T_{A}, T^{A}>
$$

where $G_{N}$ is the Newton gravitational constant, $G_{T}$ is a new constant introduced by the torsion.

The calculation shows that all the kinetic terms are present. With the mass terms in the final form of the Lagrangian, we come to the conclusion that in this version our theory of gravity in the Connes-Lott spacetime model contains the ordinary Einstein gravity, one massive non-linear tensor, one massive and one massless vector, one massive and one Brans-Dicke scalar fields.

\section{CONCLUSION}

In this paper, a new specific model of gravity in noncommutative sapcetime is proposed. However, in each step, the possible alternatives are pointed out to show how one can retain the desirable contents by a proper choice. In the final result, the solution of this paper requires to include the torsion in the final Lagrangian to retain the full spectrum of the discretized KaluzaKlein model.

From mathematical point of view, this construction has some interesting features, which deserve more discussions. As stated previously in this paper, the starting algebra $\mathcal{A}=C^{\infty}(C, \mathcal{M}) \oplus$ $C^{\infty}(C \mathcal{M})$ is too large for physical applications. The hermiticity condition on the physical 1-forms can give the correct physical content for the gauge theory. However, the set of the hermitian forms does not closed as $\mathcal{A}$-bimodule. As we shall see, our model can be based on a minimal algebra for physics.

The kinetic terms of the vielbein components must have correct signatures to be physical fields. The signature will be correct with the following restrictions on the vielbein components in the equation (17)

$$
\begin{aligned}
E_{a}^{\mu}(x) & =\left(\begin{array}{cc}
e_{+a}^{\mu}+i e_{-a}^{\mu} & 0 \\
0 & e_{+a}^{\mu}-i e_{-a}^{\mu}
\end{array}\right) \\
A_{\mu}(x) & =\left(\begin{array}{cc}
a_{+\mu}+i a_{-\mu} & 0 \\
0 & a_{+\mu}-i a_{-\mu}
\end{array}\right) \\
\Phi(x) & =\left(\begin{array}{cc}
\phi_{+}+i p h i_{-} & 0 \\
0 & \phi_{+}-i \phi_{-}
\end{array}\right),
\end{aligned}
$$

where $e_{ \pm a}^{\mu}, a_{ \pm \mu}$ and $\phi_{ \pm}$are physical real fields.

With this restriction, the theory can be constructed consistently from the subalgebra $\mathcal{A}^{\prime}$ and $\Omega^{p}\left(\mathcal{A}^{\prime}\right)$ will be $\mathcal{A}^{\prime}$-bimodule. With this algebra in the spectral triple, there is no need for additional condition on the differential forms that represent the physical fields.

The involutive operation " " on the 0 -forms determines the form inner product. This choice, in turns, is due to the decision that we make about what kind of theory one wants to obtain in the limit two sheets of spacetime become identical. The inner product defined with the operation " " on 0-forms gives kinetic terms, which are diagonal in $e_{a \pm}^{\mu}, a_{\mu \pm}$ and $\phi_{ \pm}$. In the 
limit, where two sheets of spacetime are identical, all the massive modes $e_{a-}^{\mu}, a_{\mu-}$ and $\phi_{-}$of this model vanish and one obtain the truncated Kaluza-Klein spectrum [27].

\section{ACKNOWLEDGMENT}

This work is dedicated to the memory of Victor Isacovich Ogievetsky. In our last meeting in Philadelphia in Summer 1995, my plan on noncommutative geometry has received enthusiastic encouragements from him. It is regretful that the realization of the plan has been postponed and he cannot see the results. Thanks are also due to K. C. Wali for the collaboration in the research program.

\section{REFERENCES}

[1] A. Connes, Noncommutative Geometry, (Academic Press, 1994); A. Connes, Noncommutative Differential Geometry, Publ. I.H.E.S. 62 (1986), 257; A.Connes, Noncommutative Geometry and Physics in Gravitation and Quantizations, Les Houches, Session LVII, (Elsevier Science B.V. 1995).

[2] A. Connes, Essay on Physics and Noncommutative Geometry, in The Interface of Mathematics and Particle Physics, Oxford Univ.Press (1990), 9.

[3] A. Connes and J. Lott, Nucl. Phys. Suppl. B18 (1990) 29; A. Connes and J. Lott, The Metric Aspect on Nonncommutative Geometry, in Proceedings of the 1991 Carges Summer School, ed. J. Fröhlich et al., (Plenum, 1992).

[4] A. Connes, Gravity Coupled with Matter and the Foundation of Noncommutative Geometry, hep-th/8603053 (1996).

[5] D.Kastler, Commun. Math. Phys. 166 (1995) 633.

[6] R. Coquereaux, J. Geom. Phys. 6 (1989) 425.

[7] J. Madore, An Introduction to Noncommutative Geometry and its Physical Applications, (LMS Lecture Notes 206, 1995).

[8] N. A. Viet and K. C. Wali, Intl. J. Modern Phys., A11 (1996) 2403.

[9] G. Landi An Introduction to Noncommutative Spaces and their Geometries, (Springer-Verlag, 1997).

[10] A. H. Chamseddine, G. Felder, and Fröhlich, Comm. Math. Phys. 155 (1993) 205; A. H. Chamseddine, J. Fröhlich, and O. Grandjean, J. Math. Phys. 36 (1995) 6255.

[11] W. Kalau and M. Walze, J. Geom. Phys. 16 (1995) 327.

[12] G. Landi, Nguyen Ai Viet, and K. C. Wali, Phys. Lett. B 326 (1994) 32.

[13] Nguyen Ai Viet and K. C. Wali, Intl. J. Modern Phys. A 11 (1996) 533.

[14] Nguyen Ai Viet and K. C. Wali, Matter Fields in Curved Space-Time in Theoretical High-Energy Physics MRST'2000, ed. C. R. Hagen (2000), 27.

[15] Nguyen Ai Viet and K. C. Wali, Chiral Spinors and Gauge Fields in Curved Noncommutative Space-time, hepth/0212064 (to be published).

[16] Nguyen Ai Viet Predictions of Noncommutative space-time in MRST'94 What Next? Exploring the Future of High-Energy Physics, ed.K.R.Cudel et al,(World Scientific, 1994).

[17] Nguyen Ai Viet, (To memory of E. Wigner), Heavy-Ion Phys. 1 (1995) 263.

[18] Ch. W. Misner, K. S. Thorne, and J. A. Wheeler, Gravitation, (W. H. Freeman and Company, New York, 1973).

[19] R. M. Wald General Relativity, (The University of Chicago Press, Chicago and London, 1984).

[20] M.Nakahara, Geometry, Topology and Physics, (Institute of Physics Press, 1992).

[21] T. Eguchi, P. B. Gilkey, and A. J. Hanson, Gravitation, Gauge theories and Differential Geometry Physics Reports 66 (6) (1990)

[22] M. Dubois-Viollete, Lectures on graded Differential algebras and Noncommutative Geometry LPT-ORSAY 99/100, qa/9912017 (1999).

[23] A. Sitarz, Class. Quant. Grav. 11 (1994) 2127.

[24] C. Klimcik, A.Pompos, and V.Soucek, Lett. Math. Phys. 30 (1994) 259. 
[25] Bin Chen, Takesi Saito, and Ke Wu, Prog. Theor. Phys. 92 (1994) 881; G. Konisi, Takesi Saito, and Ke Wu, Prog. Theor. Phys. 93 (1995) 621.

[26] M. Dubois-Violette, R. Kerner, and J. Madore, J. Math. Phys. 31 (1990) 316; J. Madore, Phys. Rev. D 41 (1990) 3790; M. Dubois-Violette, J. Madore, T. Masson, J. Mourad, J.Mourad, J.Math.Phys. 37 (1996), 4089; J.Madore, Class. Quant. Grav. 13 (1996) 2109; J.Mourad, Class. Quant. Grav. 12 (1995) 965.

[27] Th.Kaluza, Sitzuuza, Sitzungsber. Preuss. Akad. Wiss. Phys. Math. Klasse 966 (1921) ;O.Klein, Z.F. Physik 37 (1926) 895; Y.Thirry, Comptes Rendus (Paris) 226 (1948) 216. 